\title{
Magnetic Declination Chart 2006 of Europe - produced by the MagNetE Group
}

\author{
Gerald Duma ${ }^{1,{ }^{\star}}$, Barbara Leichter ${ }^{1}$ and the MagNetE Group ${ }^{2}$ \\ ${ }^{1}$ Central Institute for Meteorology and Geodynamics, Vienna, Austria \\ ${ }^{2}$ MagNetE Group: authors listed in chapter Map authors
}

\section{Article history}

Received September 29, 2011; accepted February 22, 2012.

Subject classification:

Main geomagnetic field, Global and regional models, Magnetic anomalies, Instruments and techniques, Data processing.

\begin{abstract}
During its $4^{\text {th }}$ Workshop in Helsinki in 2009 the Group for European Repeat Station Surveys, MagNetE, decided to produce a European Declination Chart 2006 which is based on the numerous magnetic repeat station measurements performed in more than 20 countries in Europe. All surveys were conducted within a relatively short period between 2005 and 2007, the data set consists of declination values of 382 repeat stations and the annual mean values of 42 observatories. These first accorded surveys in Europe, providing hundreds of highly accurate magnetic vector data, can be considered the first geomagnetic survey of Europe. The resulting map shows the magnetic declination, which is of main public interest for navigation purposes.
\end{abstract}

\section{Introduction}

An appropriate graphic representation of the Earth's magnetic field requires a sufficient number of data points which cover the respective region most uniformly, that is, equally spaced. Because of the fact that the geomagnetic field is continuously changing with time, it is an additional requirement that all data refer to the same point of time, otherwise the magnetic map would not represent the geographical distribution of magnetic values, but it would be a mixture of spacial and temporal differences of the magnetic field. Luckily, there exists a procedure to remove the errors of not simultaneously measured field points, at least for short time differences of up to several months. This procedure is named 'magnetic reduction' and it requires a magnetic observatory placed not too far from the survey area. The short and long period magnetic changes recorded there are considered to be the same as at the field stations in the survey area. Thus, the difference of the magnetic field values measured at the observatory and a field station at the same point of time reflects the true spatial difference which is finally visualized in a magnetic map. That is, the magnetic observatories serve as indispensable reference stations. A certain error due to this reduction procedure is unavoidable since the difference between field station and observatory is not constant but undergoes slow changes, named 'secular variation'. Consequently, the bigger the time interval between single measurements of a survey, the higher the chance of big 'reduction errors'.

Therefore, in order to obtain reliable data reflecting the instantaneous geomagnetic field in an area of continental size like Europe it was necessary to measure on ground such a huge region in a short interval of e.g. one single year. This is feasible only by a close cooperation of many European states that agree on such a dense survey schedule. Due to the MagNetE initiative such an agreement was actually obtained among the respective geomagnetic institutions in 21 states.

\section{Magnetic repeat stations}

In the second half of the $20^{\text {th }}$ century, most European states performed full geomagnetic surveys in their countries in intervals of 10 or 20 years, with high station density. The data provided the basis for the preparation of the countries' geomagnetic charts. But in addition it was necessary to regularly update those charts to provide information on the most recent values of the magnetic field, mainly for navigation purposes, in particular for aviation. Therefore, measurements at a fewer number of field stations were done which were placed evenly across a country and which could be measured in a relatively short period. Those stations are named 'magnetic repeat stations'. An overview of all magnetic surveys in European countries in the $2^{\text {nd }}$ half of the past century is given by Korte [1999].

Repeat station sites are visited more frequently, in one to five year cycles, and the measurements must provide very accurate magnetic values to determine the annual rate of magnetic changes (secular variation) at these sites precisely. For this reason, the measurements need to be done exactly at the same point and in the same instrument position at any station. The repeat station sites therefore have to be marked and stabilized very well, usually by non-mag- 

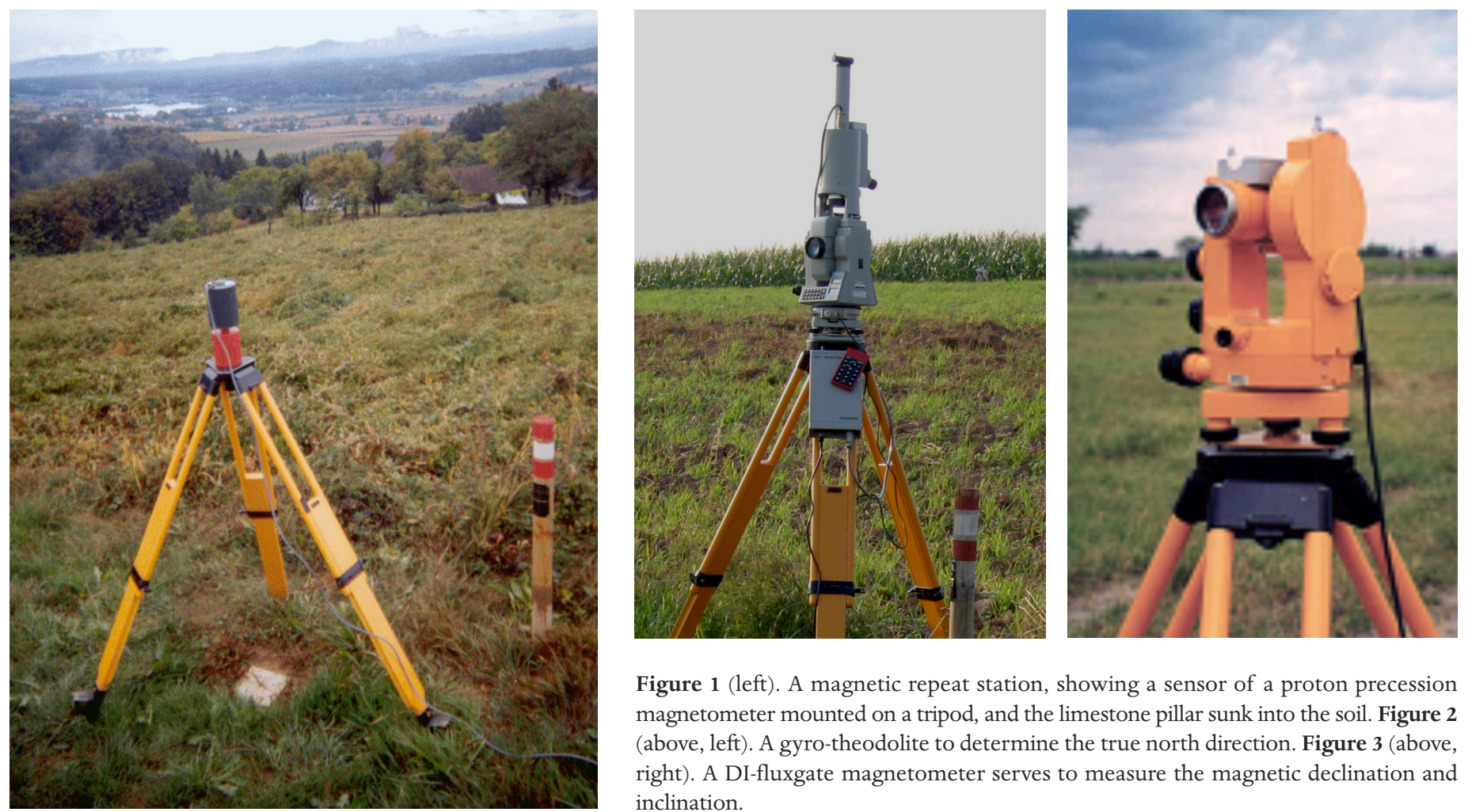

Figure 1 (left). A magnetic repeat station, showing a sensor of a proton precession magnetometer mounted on a tripod, and the limestone pillar sunk into the soil. Figure 2 (above, left). A gyro-theodolite to determine the true north direction. Figure 3 (above, right). A DI-fluxgate magnetometer serves to measure the magnetic declination and inclination.

netic concrete pillars embedded some 70 to $100 \mathrm{~cm}$ into the soil. Figure 1 shows such a site.

The most frequently used instruments for repeat station surveys are so-called DI-instruments to measure the magnetic declination (D) and inclination (I) and proton precession magnetometers for measuring the total magnetic field intensity (F). For determination of geographic North often a gyro-instrument is used, in particular if there are bad weather conditions and the azimuth reference marks in the station's vicinity are not visible due to fog. Figures 1-3 show such devices. Another frequently used method to find true North are sun-observations [e.g., Jankowski and Sucksdorff 1996], which can be performed only under clear sky conditions. The north direction at the repeat station needs to be known to determine the magnetic declination which is the angle between the vectors of horizontal intensity and geographic North.

Several countries operate a continuously recording three-component variometer as a reference station close to the repeat station site for the period of measurements. This is the most efficient survey strategy to minimize the magnetic reduction error since the magnetic variations at the field point and at the nearby variometer site coincide best.

As reported e.g. by Korte [1999] the reduction procedure for raw field measurements contributes the largest part of the error in the final repeat station data. Estimates show, that the maximum reduction error for the magnetic declination amounts to some 2.0 arcminutes and the mean reduction error to about 0.4 arcminutes. In comparison, the inaccuracy of the instruments themselves and due to measurement errors (e.g by scale reading) cause in general inac- curacies of no more than one arcminute.

A subject of scientific discussions in the MagNetE workshops was of course what should be the average distance between repeat stations and what the best period of re-occupation of the stations in order to obtain the best information on the magnetic secular changes in Europe by a minimum of survey time and expenditures. According to analyses performed by MagNetE members a station distance of 100 to $200 \mathrm{~km}$ and an interval of about 2-3 years to repeat the surveys is appropriate.

A detailed description of the procedures of measurements with different instruments at a repeat station cannot be given here because of its complexity. But a comprehensive paper on all important details of such measurements was written by Newitt et al. [1996].

\section{The data set 2006 for Europe}

Based on the plan of MagNetE to perform repeat station measurements in each country preferably every second year, that is in the even years 2004, 2006, 2008 a.s.o., most of the data were gathered in 2006 and in one year before and after, close to 2006.

An inventory showed that a data set of 382 stations in 21 countries was available. All data should be 'reduced' to the middle of the year 2006. In addition, annual mean values of 42 European geomagnetic observatories were available for 2006.

All data was acquired at the WDC for Geomagnetism, Edinburgh, British Geological Survey, and for scientific use it can be retrieved at the web address http: / / www.geomag. bgs.ac.uk/gifs/surveydata.html. 


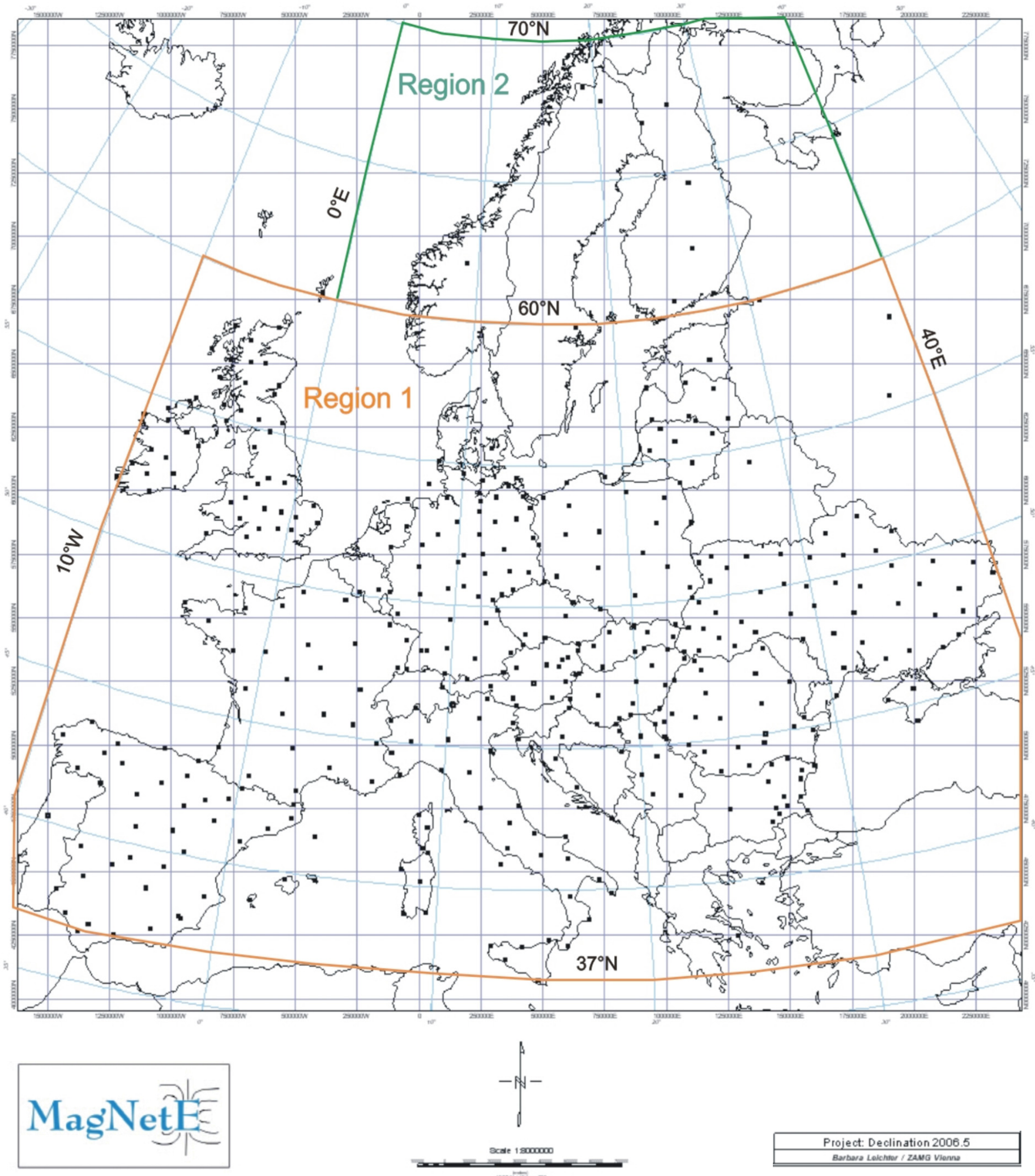

Figure 4. Magnetic Repeat Stations 2006.5. Geographical distribution of all points of measurement in 21 states, the data of which contributed to produce the European geomagnetic chart for 2006. Region 1 and Region 2 mark areas with different density of measurement sites.

The geographical positions of the repeat stations and observatories are shown in Figure 4.

We see that central and southern Europe is covered very well with stations which provide 2006 data, with an almost ideal mean station distance of some $150 \mathrm{~km}$ (Region 1). The number of data available for Region 2 at geographical lati- tudes bigger $60^{\circ} \mathrm{N}$ is rather low and a mean spacing of 450 $\mathrm{km}$ is computed. Since no ground measurements are available over the sea, like the Atlantic Ocean in the North and West and the Mediterranean Sea in the South, and sparse measurements in the East, declination values of the International Geomagnetic Reference Field, IGRF 2011 [IAGA 


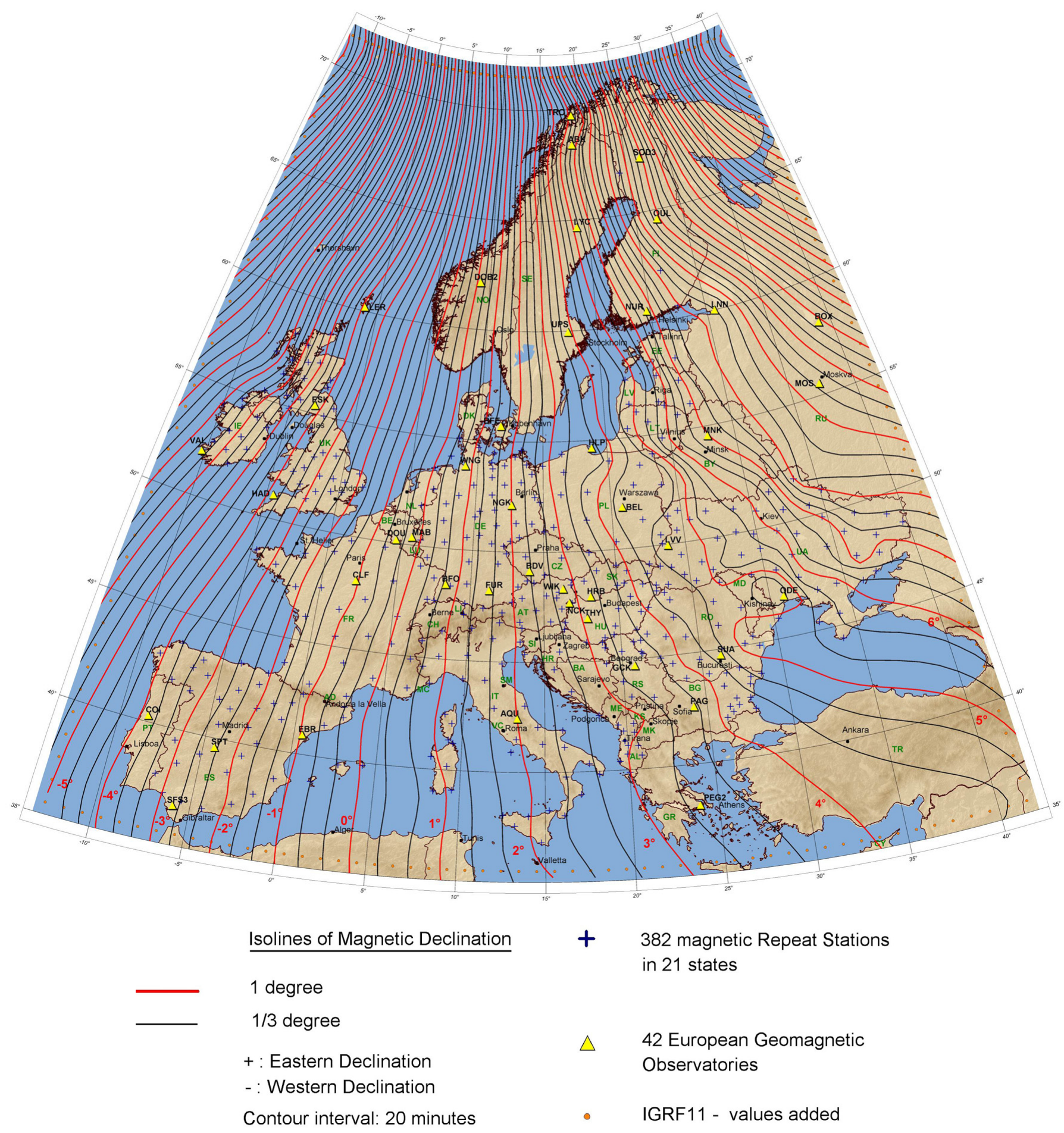

Figure 5. (Top) Magnetic Declination 2006, product of MagNetE - Magnetic Network in Europe: the final layout of the European declination 2006 map. (Bottom) Legend of the declination 2006 map.

Working Group V-MOD 2010] have been added along these map border lines (see Figure 5).

The IGRF is a mathematical approximation of the global magnetic field. It is released by the International Association of Geomagnetism and Aeronomy (IAGA, http:// www.iugg.org/IAGA). A statistic analysis shows that the standard deviation of the IGRF values from the 382 repeat station values in Europe is about 20 arcminutes for declination. But an extrapolation of the measured field in Central Europe to the IGRF points aligned at the map's borders results in much larger deviations there, up to 200 arcminutes for declination. Thus the application of IGRF values along the map edges makes the field representation realistic in those areas.

\section{The map}

The map covers an area enclosed between the meridians $13^{\circ} \mathrm{W}$ and $42^{\circ} \mathrm{E}$ and the parallels of latitude at $35^{\circ} \mathrm{N}$ and $72^{\circ} \mathrm{N}$. It represents the magnetic declination caused by the main field and large scale influences from magnetised structures in the Earth's crust. 
In order to visualize the magnetic declination field over this entire region, regularly spaced grid data have to be computed from irregularly distributed measurement points in Europe. This is done by interpolation, for which we used the Kriging method [e.g., Journel and Huijbregts 1978] . This is a statistical approach to produce a grid of declination data, which is the basis for the contouring procedure. The grid size is $100 \mathrm{~km}$ in our case. The map was plotted using the software Oasis montaj 7.2 (www.geosoft. com).

A legend on the map informs of the procedures of measurements and map production which have been applied, containing several details which all are mentioned in this paper, too.

A first draft of the map was plotted at the Central Institute for Meteorology and Geodynamics (ZAMG), Vienna. Following various suggestions from members of the MagNetE Group, a second map version was compiled in November 2010. By decision of the MagNetE Coordination Committee, the draft map had undergone a review process. Two reviewers have sent their comments, which were taken into account. Numerous suggestions were also received during the $5^{\text {th }}$ MagNetE Workshop in Rome, 2011. The Commission for the Geological Map of the World (CGMW, Paris) decided to print the map and serve for its distribution, following the proposal of M. Mandea, the IAGA Secretary General.

The map of magnetic declination 2006 in Europe in its final layout is shown in Figure 5.

In general, the isolines of declination in central Europe show a very regular course, similar to the isogones based on the IGRF 2011 model. It turned out that the measurements in central Europe (Region 1, Figure 4) deviate from the IGRF values with a standard deviation of 20 minutes, but the mean value of deviation is only some 3 arcminutes . Of course, the IGRF can not fit local or even regional magnetic anomalies since it is a mathematical function with a limited number of terms and their corresponding spherical harmonic coefficients. From Figure 5 it becomes evident, that the field in East Europe is less quiet. This applies to the NW-SE trending region, starting from the North Sea, covering East Poland, the Ukraine and the regions NW of the Black Sea. Actually, this zone also appears in the first World Digital Magnetic Anomaly Map (WDMAM), produced by an IAGA task group [Korhonen et al. 2007]. It is based on a huge amount of total intensity data from ground and marine surveys as well as from satellite measurements.

The European section of this global map is shown in Figure 6. The above mentioned zone of magnetic disturbances corresponds with the transition zone to the so-called 'Russian Platform', an older geological province (Cambrian) of the continental crust [Bubnoff 1926]. It contains rock of different magnetic properties.

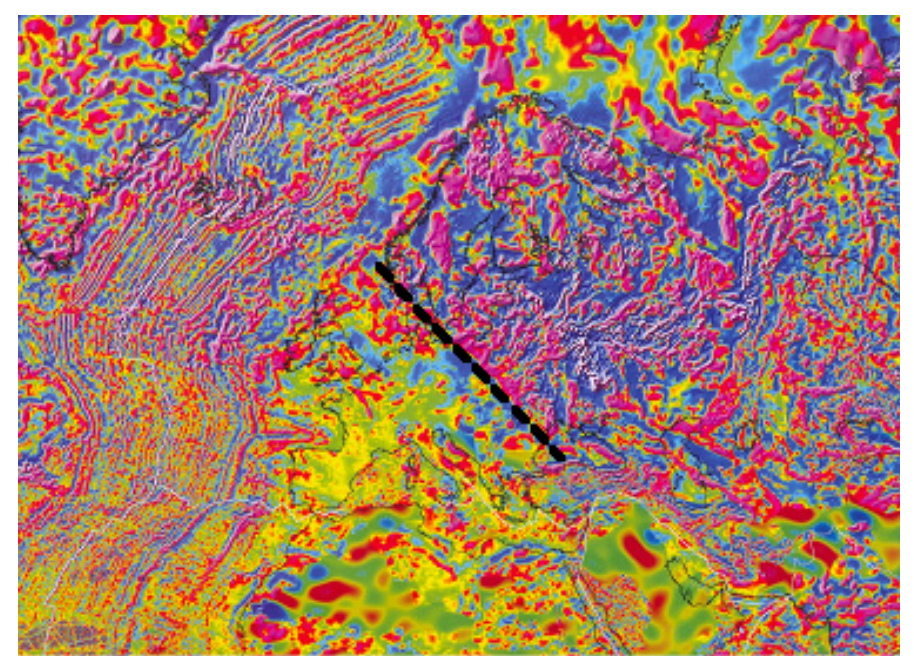

Figure 6. European section of the World Digital Magnetic Anomaly Map (WDMAM) [Korhonen et al. 2007]. The dotted line marks the transition zone to the 'Russian Platform' in the NE. This zone of changing magnetic performance turns out also from the European declination 2006 map presented in Figure 5.

In order to assess the declination values for one or two years before and after 2006, a map for the annual change (Figure 7) is displayed on the map, too, derived from the IGRF. During the year 2006 the magnetic declination changed by about +5 arcminutes in the SE of Europe (Turkey) and by some +11 arcminutes in the NW ( $\mathrm{N}$ of UK and coast of Norway). The mean annual change in central Europe was about +7 arcminutes.

\section{Access to the survey data, the map and to MagNetE}

The survey data can be retrieved from the WDC for Geomagnetism, Edinburgh, British Geological Survey, for research purposes at http:/ / www.geomag.bgs.ac.uk/gifs / surveydata.html.

The chart is printed and offered for sale by the Commission for the Geological Map of the World (CGMW, Paris): http://ccgm.free.fr .

More information about MagNetE is available under http:/ / www.gfz-potsdam.de (Find: MagNetE). Surveyors who want to participate in MagNetE activities are very welcome and may send an e-mail to magnete-subscribe@gfzpotsdam.de.

\section{Conclusions}

The joint magnetic survey activity within a small time window in 21 European states provided an accurate basis for the representation of the magnetic field 2006 in Europe.

Since the concept for the conduct of such concerted surveys, developed by MagNetE, has proved very successful, it is planned to continue this survey activity. An update of the map for 2006 is planned for the declination 2010 and a representation of the magnetic total intensity 2010, too. Thus, the plans are to make a geomagnetic map of Europe a 'periodic product'. 


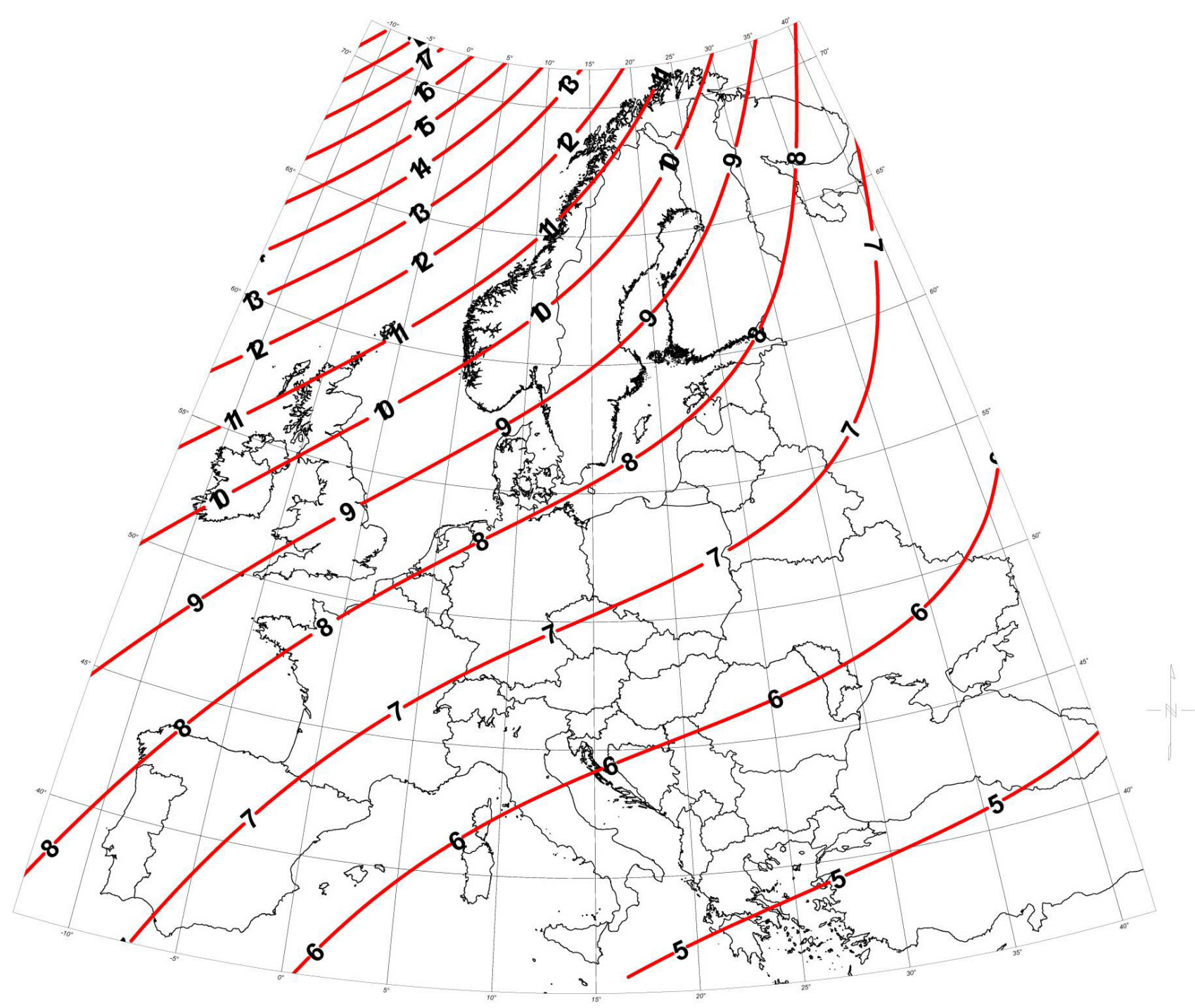

Figure 7. Annual change of declination (minutes/ per year): map of the annual change ('secular variation') of the magnetic declination in 2006.

\section{Map authors}

The map is the result of time consuming and often hard survey work done by the staffs of the national geomagnetic institutions or universities. All survey data was acquired at the WDC for Geomagnetism, Edinburgh. The final concept of map production and layout was a result of intense discussions among the MagNetE participants and the comments of two reviewers. Finally, the map was plotted at the Central Institute for Meteorology and Geodynamics, Vienna.

Table 1 lists the authors and institutions who have significantly contributed to the map project.

\section{For production:}

Duma Gerald and Leichter Barbara

Central Institute for Meteorology and Geodynamics - ZAMG, Vienna

\section{From MagNetE Group:}

Bayer Tomas

Institute of Geophysics of the ASCR, Prague

Brkic Mario

University of Zagreb, Faculty of Geodesy, Zagreb

Csontos Andras

Eötvös Loránd Geophysical Institute, Budapest

De Santis Angelo

Istituto Nazionale di Geofisica e Vulcanologia - INGV, Rome

Demetrescu Crisan

Institute of Geodynamics, Romanian Academy, Bucharest

Dobrica Venera

Institute of Geodynamics, Romanian Academy, Bucharest

Dominici Guido

Istituto Nazionale di Geofisica e Vulcanologia - INGV, Rome 


\begin{tabular}{|c|c|}
\hline Horacek Josef & Institute of Geophysics of the ASCR, Prague \\
\hline Korte Monika & Helmholtz-Zentrum, Deutsches GeoForschungsZentrum - GFZ, Potsdam \\
\hline Kultima Johannes & Finnish Meteorological Institute - FMI, Rykk \\
\hline Maksymchuk Valentyn & Subbotin's Institute of Geophysics NAS of Ukraine, Carpathian Branch, Lviv \\
\hline Mandea Mioara & Institut de Physique de Globe de Paris - IPGP, Paris \\
\hline Macmillan Susan & British Geological Survey - BGS, Edinburgh \\
\hline Orlyuk Mykhailo & Institute of Geophysics NAS of Ukraine, Kiev \\
\hline Pajunpää Kari & Finnish Meteorological Institute - FMI, Rykk \\
\hline Popeskov Dragan & Geomagnetic Institute, Grocka \\
\hline Schwarz Gerhard & Geological Survey of Sweden - SGU, Uppsala \\
\hline Shanahan Tom & British Geological Survey - BGS, Edinburgh \\
\hline Socias Isabel & Instituto Geografico Nacional, Madrid \\
\hline Srebrov Bozhidar & National Institute for Geophysics, Geodesy and Geography, Sofia \\
\hline Sugar Danijel & University of Zagreb, Faculty of Geodesy, Zagreb \\
\hline Sulakova Lubova & Latvian Geospatial Information Agency, Riga \\
\hline Thebault Erwan & Institut de Physique de Globe de Paris - IPGP, Paris \\
\hline Vaczyova Magdalena & Geophysical Institute, Slovak Academy of Science, Hurbanovo \\
\hline Valach Fridrich & Geophysical Institute, Slovak Academy of Science, Hurbanovo \\
\hline Vujic Eugen & Institute for Geomatics, University of Zagreb, Faculty of Geodesy \\
\hline Welker Elzbieta & Institute of Geodesy and Cartography, Warsaw \\
\hline
\end{tabular}

Table 1. Authors of the map and their affiliation.

\section{References}

Bubnoff, S. von (1926). Geologie von Europa, Band 1, Borntraeger Berlin.

IAGA Working Group V-MOD (2010). International Geomagnetic Reference Field: the eleventh generation, Geophys. J. Int., 183 (3), 1216-1230.

Jankowski, J., and C. Sucksdorff (1996). Guide for magnetic measurements and observatory practice, International Association of Geomagnetism and Aeronomy, Warsaw, $235 \mathrm{pp}$.

Journel, A.G., and Ch.J. Huijbregts (1978). Mining Geostatistics, Academic Press Limited, London, 597 pp.

Korhonen, J.V., J.D. Fairhead, M. Hamoudi, K. Hemant, V. Lesur, M. Mandea, S. Maus, M. Purucker, D. Ravat, T. Sazonova and E. Thebault (2007). Magnetic Anomaly Map of the World Scale 1:50,000,000, $1^{\text {st }}$ edition, Commission for the Geological Map of the World, Paris, France.

Korte, M. (1999). Kombination regionaler magnetischer Vermessungen Europas zwischen 1955 und 1995, Scientific Technical Report STR99/11, GeoForschungsZentrum Potsdam, 100 pp.

Newitt, L.R., C.E. Barton and J. Bitterly (1996). Guide for magnetic repeat station surveys, International Association of Geomagnetism and Aeronomy, Boulder, 112 pp.

^Corresponding author: Gerald Duma,

Central Institute for Meteorology and Geodynamics, Vienna, Austria; email: gerald.duma@zamg.ac.at; gerald.duma@yahoo.com.

(C) 2012 by the Istituto Nazionale di Geofisica e Vulcanologia. All rights reserved. 